SHORT REPORT

\title{
Mood changes with deep brain stimulation of STN and GPi: results of a pilot study
}

\author{
M S Okun, J Green, R Saben, R Gross, K D Foote, J L Vitek
}

J Neurol Neurosurg Psychiatry 2003;74:1584-1586

The results of this study suggest that there are mood changes associated with deep brain stimulation of the subthalamic nucleus (STN) and the globus pallidus interna (GPi). Further, optimal placement of electrodes in both STN and GPi seems to result in overall improvement in mood and is associated with a lower incidence of adverse mood effects than stimulation outside the optimal site. Preliminary data from this study, however, suggest that slight movement dorsal or ventral to the site of optimal motor performance may be associated with more adverse changes in mood with STN stimulation than with GPi stimulation.

$\mathrm{D}$ eep brain stimulation (DBS) of both the subthalamic nucleus (STN) and the globus pallidus interna (GPi) has been shown to be effective in the treatment of the cardinal motor signs of Parkinson's disease (tremor, rigidity, and bradykinesia). ${ }^{12}$ Many reports suggest more robust improvements in the motor scores of the unified Parkinson's disease rating scale (UPDRS) with STN DBS, and an ability to reduce antiparkinsonian drug treatment with STN DBS but not GPi DBS. ${ }^{2}$ Consequently the subthalamic nucleus has become the preferred target at most centres. There is, however, increasing evidence that STN DBS may be associated with a higher incidence of adverse changes in mood compared with GPi DBS. ${ }^{3-12}$ Mood is one factor that significantly influences the quality of life, and thus the characterisation of mood changes resulting from stimulation of both STN and GPi will be important in choosing the optimal target for patients undergoing DBS. To further characterise the mood changes that occur in STN and GPi DBS, we assessed mood changes associated with stimulation in nine patients who underwent either form of treatment.

\section{METHODS}

We studied nine patients with idiopathic Parkinson's disease. They were all examined by a movement disorders specialist and had at least two cardinal motor signs including akinesia/ bradykinesia, rigidity, or tremor, and three supporting signs which could include unilateral onset, rest tremor, progressive disorder, asymmetry, excellent response to levodopa, dyskinesia, levodopa response for five years, and clinical course of 10 years. Five had unilateral STN DBS and four had unilateral GPi DBS (table 1).

All were assessed on the UPDRS III motor scale (off drug treatment) and the visual analogue mood scale (VAMS) ${ }^{13}$ under five randomised conditions: off stimulation and during monopolar stimulation with each of four contacts (Medtronic 3387 DBS electrode). The UPDRS III was administered five minutes after each change of testing condition (table 2). The VAMS was administered 30 minutes after the change of testing condition. The mood scale included subscores for each of eight categories: afraid, confused, sad, angry, energetic, tired, happy, and tense.

Patient raw scores on the VAMS were converted to $\mathrm{T}$ scores using normative data provided in the test manual. Patients were screened with the Mattis dementia rating scale, the Hamilton depression scale, the Hamilton anxiety scale, and the geriatric depression scale to exclude cognitive dysfunction or active psychiatric disease before entry into the study.

Inclusion criteria for the study included an age between 30 and 75 years, a diagnosis of idiopathic Parkinson's disease, a clear response to levodopa, intractable disabling motor fluctuations, dyskinesias, or freezing episodes, a Mattis dementia rating scale score of more than 130, and no significant active psychiatric disorder. All patients were rated Hoehn and Yahr grade III or greater in the "off condition." All patients underwent the same surgical technique (multiple pass microelectrode recording, macrostimulation, target verification by postoperative magnetic resonance imaging), and signed their informed consent before participating in the study.

\section{RESULTS}

Effects of DBS on mood when stimulating at the site of optimal motor improvement

Stimulation at the site of optimal motor benefit (determined by the UPDRS III motor score) was associated with changes in mood in patients in both DBS groups. Change in mood was identified as a minimum of one standard deviation change in at least one VAMS subscale (table 3). For STN patients, four of five showed improvement on at least one subscale with optimal stimulation, and three showed improvement on at least two subscales. Two patients showed decline, each on only one subscale. For GPi patients, two of four showed improvement of at least 2 SD on four VAMS subscales when on optimised stimulation. Only one patient showed worsening, and this occurred on only one subscale (tense).

\section{Effects of DBS on mood when stimulating dorsal or ventral to the site of optimal motor benefit}

Stimulation one contact dorsal to the optimal contact led to improvement in at least two subscales in two of the five STN patients and two of the four GPi patients. Stimulation dorsally led to a decline in at least two subscales in two of the five STN patients and one of the four GPi patients. Stimulation ventrally led to no improvements on at least two subscales in each of the STN patients, and to improvement in at least two subscales in one of the four GPi patients. Stimulation one contact ventral to the optimal contact led to a decline in at least two subscales in two of the five STN patients, and two of the four GPi patients. Overall, slightly more worsening was seen in the number of subscales changed for the STN group (five subscales dorsally, seven subscales ventrally) than for the GPi group (three subscales 
Table 1 Patient characteristics

\begin{tabular}{|c|c|c|c|c|c|c|c|c|c|c|c|c|c|}
\hline & $\mathrm{N}$ & $\mathbf{F}$ & $M$ & Age (years) & Years PD & Dopamine & H\&Y & DRS & Ham-D-T & Ham-D-E & Ham-A-T & Ham-A-E & GDS \\
\hline STN & 5 & 3 & 2 & 60 (57 to 64$)$ & 14.4 & $903 \mathrm{mg}$ & 3.17 & 139 & 7.2 & 1.2 & 6.8 & 0.4 & 6.4 \\
\hline GPi & 4 & 2 & 2 & 56 (46 to 73$)$ & 14.0 & $713 \mathrm{mg}$ & 3.00 & 139 & 8.8 & 3.8 & 9 & 3 & 5.8 \\
\hline
\end{tabular}

Structured clinical interviews (DSM-IV) were undertaken on all patients: STN group: $\mathrm{n}=1$ major depression, recurrent, partial remission; $\mathrm{n}=1$ history of alcohol abuse; $n=1$ major depression single episode full remission; $n=1$ major depression recurrent full remission; $n=1$ no Axis I diagnosis; GPi group: $n=1$ major depression, recurrent, moderate; major depression, recurrent, full remission; $n=2$ no axis I diagnosis.

Dopamine, average amount of dopamine used in each group (STN 301-1550 mg; GPi 380-1260 mg); DRS, mean Mattis dementia rating scale score (range 136-142); F, female; GDS, Geriatric depression scale; GPi, globus pallidus interna; H\&Y, mean Hoehn and Yahr score in the off state; Years PD, years of Parkinson's disease symptoms; HAM-A-E, Hamilton anxiety scale etiologic score; HAM-A-T, Hamilton anxiety scale total score; HAM-D-E, Hamilton depression scale etiologic score; HAM-D-T, Hamilton depression rating scale total score; M, male; STN, subthalamic nucleus.

\begin{tabular}{|c|c|c|c|c|c|c|c|}
\hline $\begin{array}{l}\text { Surgical } \\
\text { site }\end{array}$ & $\begin{array}{l}\text { UPDRS } \\
\text { optimal }\end{array}$ & UPDRS off & $\begin{array}{l}\text { UPDRS } \\
\text { optimal/off }\end{array}$ & $\begin{array}{l}\text { UPDRS } \\
\text { dorsal }\end{array}$ & $\begin{array}{l}\text { UPDRS } \\
\text { optimal/dorsal }\end{array}$ & $\begin{array}{l}\text { UPDRS } \\
\text { ventral }\end{array}$ & $\begin{array}{l}\text { UPDRS optimal/ } \\
\text { ventral }\end{array}$ \\
\hline STN & 36.6 & 48.4 & $25.20 \%$ & 43.2 & $(-) 15.2 \%$ & 44.8 & (-) $19.4 \%$ \\
\hline GPi & 32.8 & 43.5 & $24.10 \%$ & 36.3 & $(-) 8.8 \%$ & 36 & $(-) 17.5 \%$ \\
\hline
\end{tabular}

dorsally, five subscales ventrally), although the STN group had one more patient than the GPi group (table 4). The magnitude of change in scales that worsened was slightly greater in the STN when compared with the GPi group, but the magnitude of change in scales that improved was better in the GPi group.

\section{DISCUSSION}

The results of this study suggest that there are mood changes associated with deep brain stimulation in the STN and GPi. Stimulation at optimally placed electrodes in both the STN and the GPi may result in some improvement in mood when compared with no stimulation. Stimulation dorsal or ventral to the optimal electrode may also produce mood changes. Owing to the small number of patients studied in this report, a statistically powered comparison of STN to GPi DBS cannot be done and will require a larger study. Additionally, changes measured at five minutes may not reflect maximal change in the motor scores. The timing of evaluations will require further study.

Despite a lack of randomised controlled studies to compare the relative effects of stimulation of GPi and STN, most centres currently choose stimulation of STN over GPi for the treatment of advanced Parkinson's disease. This choice is based on the reported greater improvement in motor scores with STN stimulation, as well as the ability to reduce antiparkinsonian drugs, an advantage not reported with GPi stimulation. ${ }^{12}$ More recently, reports of specific mood side effects induced by STN DBS ${ }^{34}{ }^{14}$ have raised questions about the use of this target, particularly in the elderly. Although changes in mood have been shown to occur during DBS in both the subthalamic nucleus and the globus pallidus, ${ }^{3-12}$ there are only a few anecdotal reports and case studies describing these findings. Mood changes reported in previous studies of STN DBS include depression, ${ }^{3}{ }^{12}$ pseudobulbar crying, ${ }^{8}$ mirthful laughter, ${ }^{4}$ mania, ${ }^{11}$ aggression, ${ }^{10}$ personality disorder, ${ }^{9}$ apathy, ${ }^{7}$ anxiety, ${ }^{79}$ and irritability. ${ }^{7}$ Additionally, the issue of an increased risk of suicide attempts with STN DBS has been raised recently. ${ }^{12}{ }^{15} \mathrm{GPi}$ DBS has not been reported to have as many mood side effects. $^{256}$ Apathy and abulia have been described in one patient, ${ }^{6}$ and mania ${ }^{16}$ in another.
Changes in mood and cognition during stimulation of the STN and GPi may be caused by spread of current to nonmotor (associative and limbic) portions of the STN and GPi, as well as spread to adjacent pathways mediating non-motor functions. Both the STN and GPi have motor and non-motor areas that project to cortical areas associated with motor, mood, and cognitive function, and stimulation of both nuclei has been reported to be associated with changes in mood and cognition to a varying degree. The STN $\left(158 \mathrm{~mm}^{3}\right)$ is a smaller nucleus than the GPi $\left(478 \mathrm{~mm}^{3}\right)$, and there are motor, associative, and limbic circuits as well as multiple fibre pathways (medial forebrain bundle, zona incerta, lateral hypothalamus) subserving associative and limbic functions located in a compact area within and adjacent to the STN. A larger sample size than in our pilot study will be needed to answer the question of whether there are more mood and

Table 3 Frequency of change in visual analogue mood scale subscores (STN and GPi) comparing the optimal motor condition to off stimulation

\begin{tabular}{|c|c|c|c|c|}
\hline & \multicolumn{4}{|l|}{ Mood } \\
\hline & \multicolumn{2}{|c|}{ Improved } & \multicolumn{2}{|c|}{ Worsened } \\
\hline & STN & GPi & STN & GPi \\
\hline Afraid & 1 & & & \\
\hline Confused & 1 & & 1 & \\
\hline $\begin{array}{l}\text { Sad } \\
\text { Angry }\end{array}$ & 2 & 2 & & \\
\hline Energetic & 2 & 1 & 1 & \\
\hline Tired & $\overline{1}$ & 2 & & \\
\hline Happy & 1 & 2 & & \\
\hline Tense & & 2 & & 1 \\
\hline Totals & 8 & 9 & 2 & 1 \\
\hline $1 S D$ & $N=6$ & $n=2$ & $n=1$ & $\mathrm{n}=0$ \\
\hline $2 S D$ & $\mathrm{~N}=1$ & $n=2$ & $n=1$ & $n=1$ \\
\hline $3 S D$ & $N=1$ & $\mathrm{n}=5$ & $n=0$ & $\mathrm{n}=0$ \\
\hline
\end{tabular}

"Improved" indicates a positive change when in the optimal motor condition, and "worsened" indicates a negative change.

$1 \mathrm{SD}=10-19$ point change on subscale; $2 S D=20-29$ point change on subscale; $3 \mathrm{SD}=>29$ point change on subscale; $n$, frequency of changes of that magnitude on subscales. 
Table 4 Mood changes on subscores of the VAMS stimulating dorsal to the optimal motor benefit (STN and GPi)

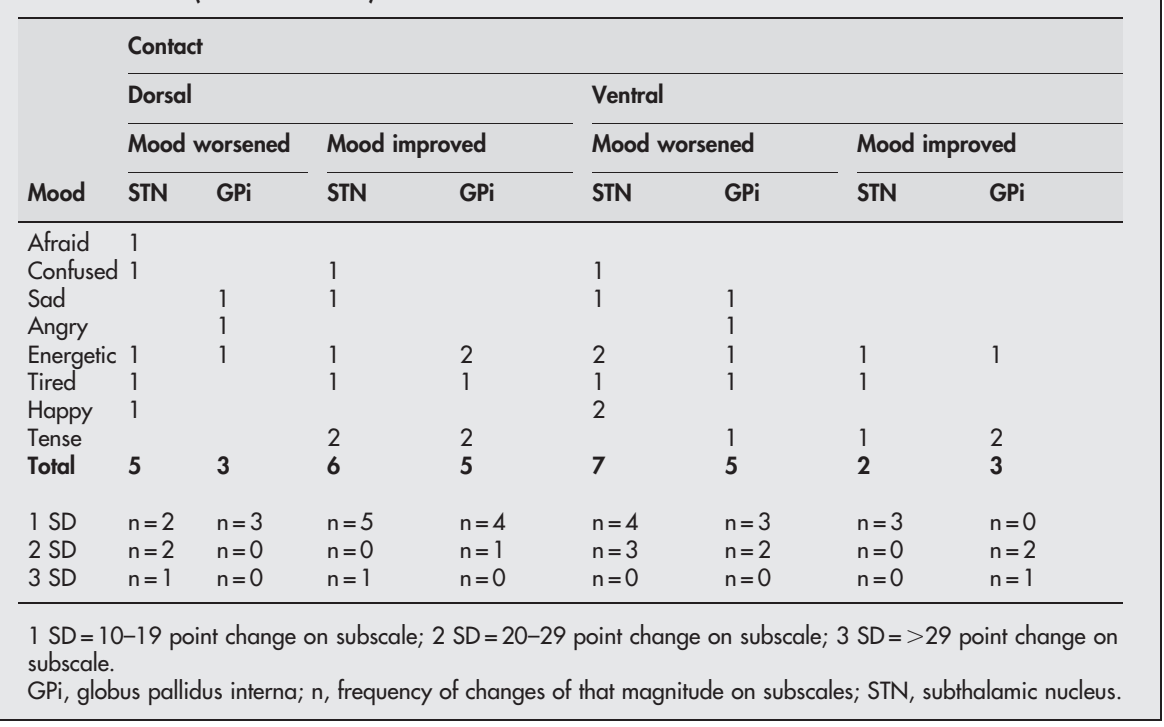

cognitive side effects with STN stimulation compared with GPi stimulation. Additionally, as shown by our small study, correctly placed leads in both the STN and the GPi are in some cases associated with positive effects on mood.

\section{Authors' affiliations}

M S Okun, K D Foote, University of Florida McKnight Brain Institute, Gainesville, Florida, USA

J Green, R Saben, J L Vitek, Emory University Department of Neurology, Atlanta, Georgia, USA

R Gross, Emory University Department of Neurosurgery

Correspondence to: Dr Michael S Okun, University of Florida McKnight Brain Institute, Department of Neurology and Neurosurgency, $100 \mathrm{~S}$ Newell Drive, Gainesville, FL 32610, USA; okun@neurology.ufl.edu

Received 31 December 2002

In revised form 26 March 2003

Accepted 2 May 2003

\section{REFERENCES}

1 Group DBSS. Deep brain stimulation of the subthalamic nucleus or the pars interna of the globus pallidus in Parkinson's disease. N Engl J Med $2001 ; 345: 956-73$.

2 Volkmann J, Sturm V, Weiss $\mathrm{P}$, et al. Bilateral high-frequency stimulation of the internal globus pallidus in advanced Parkinson's disease. Ann Neurol 1998;44(6):953-61.

3 Beijani BP, Damier P, Arnulf I, et al. Transient acute depression induced by high-frequency deep-brain stimulation [see comments]. N Engl J Med 1999:340:1476-80
4 Krack P, Kumar R, Ardouin C, et al. Mirthful laughter induced by subthalamic nucleus stimulation. Mov Disord 2001;16:867-75.

5 Troster A, Fields JA, Wilkinson SB, et al. Unilateral pallidal stimulation for Parkinson's disease: neurobehavioral functioning before and 3 months after electrode implantation. Neurology 1997:49:1078-83.

6 Dujardin K, Krystkowiak P, Defebre L, et al. A case of severe dysexecutive syndrome consecutive to chronic bilateral pallidal stimulation. Neuropsychologia 2000;38:1305-15.

7 Saint-Cyr JA, Trepanier LL, Kumar R, et al. Neuropsychological consequences of chronic bilateral stimulation of the subthalamic nucleus in Parkinson's disease. Brain 2000;123:2091-108.

8 Okun MS, Raju D, Walter BL. Reversible pseudobulbar crying from stimulation in the region of the subthalamic nucleus. J Neurol Neurosurg Psychiatry, (in press).

9 Houeto JL, Mesnage V, Mallet L, et al. Behavioural disorders, Parkinson's disease and subthalamic stimulation. J Neurol Neurosurg Psychiatry 2002:72:701-7.

10 Beijani BP, Houeto JL, Hariz M, et al. Aggressive behavior induced by intraoperative stimulation in the triangle of Sano. Neurology 2002;59:1425-7

11 Kulisevsky J, Berthier ML, Gironell A, et al. Mania following deep brain stimulation for Parkinson's disease. Neurology 2002:59:1421-4.

12 Berney A, Vingerhoets F, Perrin A, et al. Effect on mood of subthalamic DBS for Parkinson's disease: a consecutive series of 24 patients. Neurology 2002;59: 1427-9.

13 Stern RA. Assessment of mood states in neurodegenerative disease: methodological issues and diagnostic recommendations. Semin Clin Neuropsychiatry 1996;1:315-24.

14 Troster Al, Fields JA, Wilkinson SB, et al. Unilateral pallidal stimulation for Parkinson's disease: neurobehavioral functioning before and 3 months after electrode implantation. Neurology 1997;49:1078-83.

15 Doshi PK, Chhaya N, Bhatt MH. Depression leading to attempted suicide after bilateral subthalamic nucleus stimulation for Parkinson's disease. Mov Disord 2002;17:1084-5.

16 Miyawaki E, Perlmutter JS, Troster Al, et al. The behavioral complications of pallidal stimulation: a case report. Brain Cogn 2000;42:417-34. 